\title{
RATIONAL POINTS ON QUARTICS
}

\author{
January 25, 2006
}

\author{
Joe Harris \\ Mathematics department, Harvard University, \\ 1 Oxford st., Cambridge MA 02138, USA \\ harris@math.harvard.edu
}

\section{Yuri Tschinkel}

Mathematics department, University of Illinois at Chicago, Chicago, IL 60607, USA

yuri@math.uic.edu 


\section{Contents}

1 Introduction 2

2 Fano Threefolds $\quad 6$

3 A Chow ring calculation 9

4 The argument via monodromy 14

4.1 Generalities about quartic K3's . . . . . . . . . . . . 15

4.2 Analysis of the points of $C_{H} \cap L \ldots \ldots$. . . . . . . 17

5 Rational points on quartic surfaces $\quad 20$

6 An example: the Fermat quartic 24

$\begin{array}{lll}7 & \text { Quartic threefolds } & 26\end{array}$

8 Other elliptic surfaces $\quad 29$

\section{Introduction}

Of all the possible extensions to higher dimensions of Faltings's theorem, probably the most fundamental is the

Conjecture 1.1 (The Weak Lang Conjecture) Let $X$ be a variety defined over a number field $K$. If $X$ is of general type, then the set $X(K)$ of $K$-rational points of $X$ is not Zariski dense.

(While the name "Weak Lang Conjecture" has become standard usagein part to distinguish it from the "Strong Lang Conjecture" below - we should point out that as stated here it was first ventured by Bombieri for surfaces (see for example [27]) and by Vojta in [31].) 
We ask now whether a converse to this statement might hold. As it stands, the converse to the Weak Lang Conjecture cannot possibly be true. For example, if we take the product $X=\mathbb{P}^{1} \times C$ of a rational curve and a curve $C$ of genus $g \geq 2$, we get a surface that is not of general type; but by Faltings's theorem the rational points of $X$ must lie in a finite union of fibers of $X$ over $C$.

The point is that the Kodaira dimension of a variety is not a sufficiently sensitive measure of the positivity or negativity of its canonical bundle. One possible modification, if we hope to have a plausible converse to the Weak Lang Conjecture, would be to replace the hypothesis " $X$ is of general type" with " $X$ admits a dominant rational map to a positive-dimensional variety of general type"; or, given the counterexamples to this found by ColliotThélène, Skorobogatov and Swinnerton-Dyer ([10]), with "there exists a finite étale cover $Y \rightarrow X$ and a dominant rational map $Y \rightarrow Z$ to a positivedimensional variety of general type". In other words, we may make the following conjecture, which was suggested to us to by Dan Abramovich and Jean-Louis Colliot-Thélène:

Conjecture 1.2 Let $X$ be a smooth, connected projective variety defined over a number field $K$. There exists a finite extension $K^{\prime}$ of $K$ such that the set $X\left(K^{\prime}\right)$ of $K^{\prime}$-rational points of $X$ is Zariski dense if and only if no finite étale cover $Y \rightarrow X$ admits a dominant rational map $Y \rightarrow Z$ to a positive-dimensional variety of general type.

Alternatively, we can give up trying to find an if-and-only-if statement, and simply ask what sort of condition on the canonical bundle of $X$ will ensure that $X$ has a Zariski dense collection of rational points over some finite extension of $K$ : for example, we may make the

Conjecture 1.3 Let $X$ be a smooth, connected projective variety defined over a number field $K$. If the canonical bundle $K_{X}$ of $X$ is negative (that $i s,-K_{X}$ is ample), then for some finite extension $K^{\prime}$ of $K$ the set $X\left(K^{\prime}\right)$ of $K^{\prime}$-rational points of $X$ is Zariski dense.

This conjecture is easily seen to be true for curves and surfaces, where the hypothesis ensures that $X$ is rational. The first real test cases are thus Fano threefolds. In this paper, we will examine the available evidence for this 
conjecture, and add to it by analyzing one further class of Fano threefolds, the smooth quartic hypersurfaces in $\mathbb{P}^{4}$. Specifically, we will prove the

Theorem 1.4 Let $S \subset \mathbb{P}^{n}$ be a smooth quartic hypersurface defined over a number field $K$. If $n \geq 4$, then for some finite extension $K^{\prime}$ of $K$ the set $S\left(K^{\prime}\right)$ of $K^{\prime}$-rational points of $S$ is Zariski dense.

In section 7 we will show that theorem 1.4 follows as a straightforward corollary of a result about quartic surfaces:

Theorem 1.5 Let $S \subset \mathbb{P}^{3}$ be a smooth quartic surface defined over a number field $K$, and $L$ a line in $\mathbb{P}^{3}$ contained in $S$, likewise defined over $K$. Then

a. for some finite extension $K^{\prime}$ of $K$ the set $S\left(K^{\prime}\right)$ of $K^{\prime}$-rational points of $S$ is Zariski dense; and

$b$. if we assume further that $L$ does not meet six or more other lines contained in $S$, then in fact the set $S(K)$ of $K$-rational points of $S$ is Zariski dense.

Our proof of Theorem 1.5 is based on an analysis of the fibration of $S$ over $\mathbb{P}^{1}$ given by projection from the line $L$, and of the trisection of $S \rightarrow \mathbb{P}^{1}$ given by the points of $L$ itself. For any plane $H \subset \mathbb{P}^{3}$ containing $L$, let $C_{H} \subset H$ be the cubic residual to $L$ in the intersection of $H$ with $S$. The key point in our argument has to do with the relation (or lack thereof) between the points $p$ of intersection of the curves $C_{H}$ with $L$ and the hyperplane class in $\operatorname{Pic}\left(C_{H}\right)$ : the basic result, which we will establish subject to various hypotheses in the following sections, states that for all but finitely many $H$ and any point $p \in C_{H} \cap L$, the classes of $p$ and the line bundle $\mathcal{O}_{C_{p}}(1)$ are linearly independent in $\operatorname{Pic}\left(C_{p}\right)$; that is, no multiple of the point $p$ is linearly equivalent to any multiple of the hyperplane class on $C_{H}$. This will imply the desired density of rational points - it suffices to pull back the elliptic fibration to $L$. The new fibration $S^{\prime} \rightarrow L$ has a section of infinite order and by a result of Néron rational points are Zariski dense on $S^{\prime}$ and consequently on $S$.

It is worth mentioning that Conjecture 1.3 is not the strongest possible converse to the Weak Lang Conjecture. It may well be that we don't need the canonical bundle to be negative - as the Theorem 1.5 shows - but 
only nonpositive in a suitable sense. Thus, for example, we could make the stronger

Conjecture 1.6 Let $X$ be a smooth projective variety defined over a number field $K$. If the anticanonical bundle $-K_{X}$ of $X$ is nef (that is, $-K_{X}$ has nonnegative degree on every curve $C \subset X)$, then for some finite extension $K^{\prime}$ of $K$ the set $X\left(K^{\prime}\right)$ of $K^{\prime}$-rational points of $X$ is Zariski dense.

The first interesting test case for this conjecture would be K3 surfaces. In fact, we will prove it for a large class of K3 surfaces, but the question remains open for general K3 surfaces defined over a number field ${ }^{1}$.

We should also mention here the Strong Lang Conjecture - that is, the

Conjecture 1.7 (The Strong Lang Conjecture) Let $X$ be a variety defined over a number field $K$. If $X$ is of general type, then there exists a proper subvariety $\Sigma \varsubsetneqq X$ such that for any finite extension $K^{\prime}$ of $K$,

$$
\#(X \backslash \Sigma)\left(K^{\prime}\right)<\infty
$$

that is, the set of $K^{\prime}$-rational points of $X$ lying outside of $\Sigma$ is finite.

The converse to this statement seems plausible. For one thing, if $\phi: A \rightarrow$ $X$ is any nonconstant map from a rational or abelian variety to $X$, it's not hard to see that the image of $\phi$ will have to be contained in the Langian exceptional subvariety $\Sigma$. Moreover, as a consequence of the theorem of Kollár-Miyaoka-Mori varieties which are not of general type can be thought of as triple fibrations $X \rightarrow Y \rightarrow Z$, where the generic fiber of $X \rightarrow Y$ is rationally connected, $Y \rightarrow Z$ is a fibration with generic fiber of Kodaira dimension 0 and the base has Kodaira dimension $\geq 0$ (see [20], [7] and [11]). Thus the converse to the Strong Lang Conjecture hinges on whether a variety of Kodaira dimension 0 possesses a dense collection of images of rational and/or abelian varieties. Since this is known for curves and surfaces, the converse to the Strong Lang Conjecture is likewise known for all curves and surfaces, and for all threefolds except for Calabi-Yau threefolds, which represent the first real test. In general, however, it remains very much open.

\footnotetext{
${ }^{1}$ Based on ideas of the present paper Bogomolov and the second author have recently proved the conjecture 1.6 for Enriques, elliptic K3 surfaces and K3 surfaces with infinite automorphism groups [4], [5].
} 
It is also worth mentioning that there are conjectures describing asymptotics for the number of rational points of bounded height. For example, let us consider a smooth quartic hypersurface in $\mathbb{P}^{4}$. Then it is expected that the number of rational points contained in some appropriate Zariski open subset and defined over a sufficiently large finite extension of the groundfield of bounded height (induced from a standard height on $\mathbb{P}^{4}$ ) grows linearly with the height (cf. [13]).

\section{Fano Threefolds}

In this section we give a brief survey of known classification and rationality results for Fano threefolds over an algebraically closed field of characteristic zero (cf. [24], [25], [3], [21]). More details and references can be found in the recent book [19]. For our purposes it suffices to consider minimal Fano threefolds (not isomorphic to a blow up of a Fano variety).

The main invariants of Fano threefolds are: $r(X)$ - the index, defined as the maximal $r \in \mathbb{Z}$ such that $-K_{X}=r L$ for some $L$ in the Picard group $\operatorname{Pic}(X) ; \rho(X)$ - the rank of $\operatorname{Pic}(X)$ and the normalized degree $\delta(X)=$ $\left(-K_{X}\right)^{3} / r(X)^{3}$.

\section{Group I: $r \geq 2, \rho=1$}

1. $\mathbb{P}^{3}(r=4)$

2. $Q^{3}$ is the nonsingular quadric hypersurface in $\mathbb{P}^{4}(r=3)$

The remaining 5 families have $r=2$. They are indexed by $\delta$. Let $H$ be a line bundle such that $|2 H|=\left|-K_{V}\right|$.

3.

$$
\phi_{H}: V_{1} \rightarrow \mathbb{P}^{2}
$$

is a rational map with one indeterminacy point and irreducible elliptic fibers. $V_{1}$ can be realized as a double cover of the Veronese cone in $\mathbb{P}^{6}$ whose branch locus is a smooth intersection of this cone and a 
cubic hypersuface not passing through the vertex of the cone. Another realization is as a hypersurface of degree 6 in the weighted projective space $\mathbb{P}(1,1,1,2,3)$. The general $V_{1}$ is non-rational. Unirationality is unknown.

4.

$$
\phi_{H}: V_{2} \rightarrow \mathbb{P}^{3}
$$

is a double covering ramified along a smooth quartic surface. All are unirational, the general $V_{2}$ is non-rational.

5.

$$
\phi_{H}: V_{3} \rightarrow \mathbb{P}^{4}
$$

is a smooth cubic hypersurface. All are unirational, all are non-rational.

6.

$$
\phi_{H}: V_{4} \rightarrow \mathbb{P}^{5}
$$

is a smooth intersection of two quadrics. All are rational.

7. $V_{5}$ is birational to a smooth quadric $Q_{3}$.

\section{Group II: $r=1, \rho=1$}

1.

$$
\phi_{-K_{V}}: W_{2} \rightarrow \mathbb{P}^{3}
$$

is a double covering ramified along a smooth sextic surface. They are non-rational, unirationality is unknown.

2.

$$
\phi_{-K_{V}}: W_{4} \rightarrow \mathbb{P}^{4}
$$

is a smooth quartic. All are non-rational, some are unirational. In general, unirationality is unknown.

3.

$$
\phi_{-K_{V}}: W_{6} \rightarrow \mathbb{P}^{5}
$$

is a smooth complete intersection of a quadric and a cubic. All are unirational and non-rational. 
4.

$$
\phi_{-K_{V}}: W_{8} \rightarrow \mathbb{P}^{6}
$$

is a smooth complete intersection of 3 quadrics. All are unirational and non-rational.

5. $W_{10}$ are all unirational, rationality is unknown: the general one is nonrational. Geometrically, it is a section of $\operatorname{Gr}(2,5)$ in its Plücker embedding by a subspace of codimension 2 and a quadric.

6. $W_{12}, W_{16}, W_{18}, W_{22}$ are all rational.

7. $W_{14}$ are birational to a cubic threefold: all unirational, all non-rational.

\section{Group III: $\rho=2,3$}

Theorem 2.1 ( [24], p. 104) If $\rho(X)=3$, then $X$ is a conic bundle over $\mathbb{P}^{1} \times \mathbb{P}^{1}$ and has either a horizontal divisor $D \simeq \mathbb{P}^{1} \times \mathbb{P}^{1}$ or another conic bundle structure over $\mathbb{P}^{1} \times \mathbb{P}^{1}$. In particular, all varieties in this group are unirational. Non-rational varieties are pointed out in the list below.

There are 4 types of minimal Fano threefolds with $\rho=3$ :

1. Double cover of $\mathbb{P}^{1} \times \mathbb{P}^{1} \times \mathbb{P}^{1}$ ramified in a $(2,2,2)$-divisor. Non-rational.

2. Smooth member of $\left|L^{\otimes 2} \otimes_{\mathcal{O}_{\mathbb{P}^{1} \times \mathbb{P}^{1}}} \mathcal{O}(2,3)\right|$ on $\mathbb{P}_{\mathbb{P}^{1} \times \mathbb{P}^{1}}\left(\mathcal{O} \oplus \mathcal{O}(-1,-1)^{\oplus 2}\right)$ such that $X \cap Y$ is irreducible. Here $L$ is the tautological line bundle and $Y \in|L|$.

3. $\mathbb{P}^{1} \times \mathbb{P}^{1} \times \mathbb{P}^{1}$.

4. $\mathbb{P}_{\mathbb{P}^{1} \times \mathbb{P}^{1}}(\mathcal{O} \oplus \mathcal{O}(1,1))$.

The remaining varieties in this group have Picard number $\rho=2$. All are conic bundles over $\mathbb{P}^{2}$.

5. Double cover of $\mathbb{P}_{*}^{3}\left(=\mathbb{P}^{3}\right.$ blown up in one point), ramified in a divisor in $\left|-K_{\mathbb{P}_{*}^{3}}\right|$, non-rational.

6. Double cover of $\mathbb{P}^{1} \times \mathbb{P}^{2}$ ramified in a $(2,2)$-divisor. 
7. Double cover of $\mathbb{P}^{1} \times \mathbb{P}^{2}$ ramified in a $(2,4)$-divisor, non-rational.

8. Hypersurface of bidegree $(2,2)$ in $\mathbb{P}^{2} \times \mathbb{P}^{2}$, non-rational.

9. Hypersurface of bidegree $(1,2)$ in $\mathbb{P}^{2} \times \mathbb{P}^{2}$.

10. Hypersurface of bidegree $(1,1)$ in $\mathbb{P}^{2} \times \mathbb{P}^{2}$.

11. $\mathbb{P}^{1} \times \mathbb{P}^{2}$.

12. $\mathbb{P}_{\mathbb{P}^{2}}(\mathcal{O} \oplus \mathcal{O}(2))$.

13. $\mathbb{P}_{\mathbb{P}^{2}}(\mathcal{O} \oplus \mathcal{O}(1))$.

There are many more forms of Fano varieties over non-closed fields. The main result of this paper together with the above classification implies: If $X$ is a Fano variety defined over some number field $K$ which over $\mathbb{C}$ is not isomorphic to (a blow up of) $V_{1}^{2}$ or $W_{2}$, then there exists a finite extension $K^{\prime} / K$ such that the set $X\left(K^{\prime}\right)$ is Zariski dense.

One may ask for conditions which insure unirationality. Already for (minimal) Del Pezzo surfaces of degree 1 (that is, smooth minimal over $K$ surfaces $S$ with ample anticanonical bundle and $K_{S}^{2}=1$ ) it is unknown whether or not rational points are Zariski dense.

\section{A Chow ring calculation}

We use the notations of the introduction: $S \subset \mathbb{P}^{3}$ is a smooth quartic surface containing a line $L$. We consider hyperplanes $H \subset \mathbb{P}^{3}$ passing through $L$ and we denote by $C_{H}$ the cubic curve residual to $L$ in the intersection of $H$ with $S$-that is, $S \cdot H=L+C_{H}$ as divisors on $H$-and let $D_{H}=C_{H} \cap L$ be the intersection of $C_{H}$ with the line $L$. Note that projection from the line $L \subset \mathbb{P}^{3}$ gives a regular map $\pi$ from the surface $S$ to the line $M \cong \mathbb{P}^{1}$ parametrizing planes through $L$, and that the curves $C_{H}$ are simply the fibers of this map.

Note also that for any point $p \in L$, the point $p$ will lie in $C_{H}$ if and only if $H$ is the tangent plane to $S$ at $p$. Thus the restriction of the map $\pi: S \rightarrow M$ to $L \subset S$ is simply the restriction to $L$ of the Gauss map on $S$, mapping

\footnotetext{
${ }^{2}$ This case was treated in the recent paper [6].
} 
$L$ onto the line in $\left(\mathbb{P}^{3}\right)^{*}$ dual to $L$. Since the general plane $H$ containing $L$ is tangent to $S$ at the three points of $D_{H}$, this map has degree 3 . The divisors $D_{H}$ are the fibers of the restriction of this map, and so in particular the divisors $D_{H}$ form a linear system on $L$ of degree 3 .

Similarly, for any point $p \in L$, let $\mathbb{T}_{p} S \subset \mathbb{P}^{3}$ be the tangent plane to $S$ at $p$, and let $C_{p}=C_{\mathbb{T}_{p} S} \subset \mathbb{T}_{p} S$ be the cubic residual to $L$ in the intersection of $\mathbb{T}_{p} S$ with $S$. Let $D_{p}=D_{\mathbb{T}_{p} S}=C_{p} \cap L$ be the intersection with $L$; note that $p \in D_{p}$ tautologously.

We will begin by establishing a weak form of our basic result: we show that if $L$ meets no other line of $S$, then for general $H \supset L$ the points of $C_{H} \cap L$ are not rationally related to $\mathcal{O}_{C_{H}}(1)$ in $\operatorname{Pic}\left(C_{H}\right)$. The proof is a relatively elementary argument using a calculation in the Néron-Severi group of an associated surface. In the following section, we will give a more refined analysis, which will allow us to conclude the same statement subject only to the weaker hypothesis that $L$ does not meet six or more lines of $S$; while the present argument will be superseded by that one, the argument here is useful for its (relative) simplicity and applications to similar situations. (We will see some of these in section 8.)

Theorem 3.1 Assume that no other lines lying on $S$ meet L. For all but countably many points $p \in L(\mathbb{C})$, the classes of $p$ and the line bundle $\mathcal{O}_{C_{p}}(1)$ are linearly independent in $\operatorname{Pic}\left(C_{p}\right)$; that is, for any positive integer $n$ we have

$$
3 n \cdot p \not \mathcal{O}_{C_{p}}(n)
$$

Proof. We begin by introducing a basic surface associated to this configuration: the incidence correspondence

$$
T=\left\{(p, q): q \in C_{p}\right\} \subset L \times S .
$$

To see $T$ more clearly, note first that projection from the line $L$ gives a regular map $\phi: S \rightarrow M$ of $S$ to the line $M \cong \mathbb{P}^{1}$ parametrizing the pencil of planes containing $L$; the curves $C_{H}$ are the fibers of this map. Similarly, the divisors cut on $L$ by the curves $C_{H}$ form a base-point-free pencil of degree 3 on $L$; the 
restriction $\phi^{\prime}=\left.\phi\right|_{L}$ is the map associated to this pencil and correspondingly has degree 3. In these terms, the surface $T$ is simply the fiber product

$$
T=L \times_{M} S
$$

In particular, $T$ is a three-sheeted cover of $S$, branched over the union of the fibers $C_{H}$ of $S \rightarrow M$ such that $C_{H}$ is tangent to $L$. Note that for a general pair $(S, L)$ the surface $T$ will be smooth, but it need not be always: it will be singular exactly when some curve $C_{H}$ is simultaneously singular and not transversal to $L$. At worst, however, it will have isolated singularities, since by the hypothesis that $L$ meets no other lines lying on $S$ no curve $C_{H}$ can have a multiple component.

Note that $T \rightarrow L$ has a tautologous section

$$
\Sigma=\{(p, p): p \in L\} \subset T
$$

this is just the intersection of $T=L \times_{M} S \subset S \times{ }_{M} S$ with the diagonal $\Delta \subset S \times_{M} S$. As a divisor, the pullback $\nu^{*}(L)$ of the line $L$ under the three-sheeted covering $\nu: T \rightarrow S$ is thus a sum

$$
\nu^{*}(L)=\Sigma+R
$$

with $R \subset T$ flat of degree 2 over $L$. Note that since the divisors $D_{H}$ form a base-point-free linear series, the general divisor $D_{H}$ is reduced; in particular, $R$ does not contain $\Sigma$. The curve $R$ will be reducible if and only if the covering $L \rightarrow M$ is cyclic.

Now, suppose that the conclusion of our Theorem is false: that is, for some $n$, we have

$$
3 n \cdot p \sim \mathcal{O}_{C_{p}}(n)
$$

for all $p \in L(\mathbb{C})$ with $C_{p}$ smooth. Fixing a plane $\Gamma \subset \mathbb{P}^{3}$, there is thus for all but finitely many $p$ a rational function on $C_{p}$ with a pole of order $3 n$ at $p$ and zeroes of order $n$ at the points of intersection $\Gamma \cap C_{p}$, and which is nonzero and regular everywhere else. It follows in turn that there is a rational function $f$ on $T$ with divisor

$$
(f)=-3 n \cdot \Sigma+n \cdot \nu^{*} \Gamma+D
$$


where $D$ is supported on a finite union of fibers of $T \rightarrow L$. Since the hypothesis that $L$ meets no other line of $S$ insures that all fibers of $T \rightarrow L$ are irreducible, $D$ must consist of a sum of fibers $C_{p}$ of $T \rightarrow L$. Since all fibers of $T \rightarrow L$ are linearly equivalent, our theorem will thus follow from the

Lemma 3.2 The classes $\sigma, \gamma$ and $\phi \in A_{1}(T)$ of the divisors $\Sigma, \nu^{*} \Gamma$ and $C$ are independent in the group $A_{1}(T)$ of Weil divisors mod linear equivalence on $T$.

Proof. We need to begin with a basic fact (due to Mumford), whose proof is mapped out in Fulton, Examples 7.1 .16 and 8.3.11 [14].

Lemma 3.3 Let $T$ be a reduced, irreducible, normal, projective surface. We may define, for every point $p \in T$ and Weil divisors $D, E \in Z_{1}(T)$ whose supports have no common component in a neighborhood of $p$, an intersection multiplicity

$$
j(p, D \cdot E) \in \mathbb{Q},
$$

bilinear in $D$ and $E$, and a bilinear intersection pairing

$$
(\cdot, \cdot): A_{1}(T) \times A_{1}(T) \longrightarrow \mathbb{Q}
$$

on the group $A_{1}(T)$ of Weil divisors on $T$ mod rational equivalence, with the following properties:

1. If $D$ and $E$ are effective and both contain $p$, then $j(p, D \cdot E)>0$.

2. If $D$ is locally principal at $p$, that is, $D=(f)$ for some rational function $f$ in a neighborhood of $p$, and $E$ is effective and irreducible, then

$$
j(p, D \cdot E)=\operatorname{ord}_{p}\left(\left.f\right|_{E}\right) .
$$

3. If $D$ and $E$ have no common components, then

$$
([D] \cdot[E])=\sum_{p \in D \cap E} j(p, D \cdot E) .
$$


Note that by the second and third properties, the intersection pairing extends the product on the group $\operatorname{Pic}(T)$.

We may now establish Lemma 3.2 by calculating the matrix of intersection products of the classes $\sigma, \gamma$ and $\phi \in A_{1}(T)$ and showing that this matrix is nonsingular. All but one of these numbers are readily calculated. To begin with, $\phi$ is the class of a fiber of the map $T \rightarrow L$, so of course $\phi^{2}=0$; and inasmuch as $\sigma$ is the class of a section $\Sigma$ of that map, we have $(\phi \cdot \sigma)=1$. Next, $\gamma$ is the pullback of the hyperplane class under the map $\nu: T \rightarrow S \hookrightarrow \mathbb{P}^{3}$; since the map $T \rightarrow S$ has degree 3 , we have

$$
\gamma^{2}=3 \cdot \operatorname{deg}(S)=12 .
$$

Since the curves $C_{p}$ map forward to plane cubics under the map $\nu$, moreover, we have

$$
(\gamma \cdot \phi)=3
$$

and similarly, since the curve $\Sigma$ maps one-to-one onto the line $L \subset S$,

$$
(\gamma \cdot \sigma)=1 .
$$

In sum, then, we have the following table of intersection products

\begin{tabular}{r|r|r|r} 
& $\gamma$ & $\phi$ & $\sigma$ \\
\hline$\gamma$ & 12 & 3 & 1 \\
\hline$\phi$ & 3 & 0 & 1 \\
\hline$\sigma$ & 1 & 1 & $\sigma^{2}$ \\
\hline
\end{tabular}

The only mystery is the self-intersection $\sigma^{2}$ of the curve $\Sigma$ on $T$. To find this, we use a relation of linear equivalence between $\Sigma$ and a curve not containing $\Sigma$ : as we saw above,

$$
\nu^{*} L=\Sigma+R
$$

so that if $\rho=[R] \in A_{1}(T)$ is the class of $R$, we have

$$
\sigma^{2}=\left(\sigma \cdot\left[\nu^{*} L\right]-\rho\right) .
$$

Now, by the projection formula,

$$
\left(\Sigma \cdot \nu^{*} L\right)_{T}=\left(\nu_{*} \Sigma \cdot L\right)_{S}=(L \cdot L)_{S}=-2
$$


and so

$$
\sigma^{2}=-2-(\sigma \cdot \rho) .
$$

Alternatively, if we choose the plane $\Gamma \subset \mathbb{P}^{3}$ to contain $L$, we see that the inverse image in $T$ will consist of $\Sigma, R$ and the three fibers of the map $T \rightarrow L$ over the points of intersection of $C_{\Gamma}$ with $L$. Thus

$$
\gamma=\sigma+\rho+3 \phi
$$

and

$$
\sigma^{2}=(\sigma \cdot \gamma-3 \phi-\rho)=-2-(\sigma \cdot \rho) .
$$

At this point, we may readily complete the calculation for general $S$ and $L$ : in general, $T$ will be smooth, and $\Sigma$ and $R$ will intersect transversely over the points $p$ where $C_{p}$ is tangent to $L$ at $p$. The curves $C_{H}$ cut out a general pencil of degree 3 on $L$, which by Riemann-Hurwitz will have 4 branch points; thus $(\Sigma \cdot R)=4$. For arbitrary $S$ and $L$, however, $T$ may be singular at the points of intersection of $\Sigma$ with $R$-it will be so exactly when a curve $C_{H}$ has a singularity at a point of $L$-and we can no longer say precisely what the intersection multiplicity is. All we do know, in fact, is that $\Sigma$ must meet $R$ somewhere, so that

$$
(\Sigma \cdot R)>0
$$

and correspondingly

$$
\sigma^{2}<-2 .
$$

Now, we may calculate the determinant of the matrix of pairwise intersection of the classes $\sigma, \gamma$ and $\phi$ : it is

$$
-12+3-9 \sigma^{2}+3=-6-9 \sigma^{2}>0 .
$$

The matrix is thus nonsingular, the classes $\sigma, \gamma$ and $\phi$ are independent, and Lemma 3.2 is proved.

\section{The argument via monodromy}

A finer analysis of the fibration $\pi: S \rightarrow M \cong \mathbb{P}^{1}$, and specifically of the monodromy of the family on the torsion points in the Jacobian of the general fiber, yields a stronger result. 


\subsection{Generalities about quartic K3's}

To begin, we recall some basic facts about the fibration $S \rightarrow M$. First, since $S$ is smooth, the Gauss map $\mathcal{G}: S \rightarrow\left(\mathbb{P}^{3}\right)^{*}$ is regular, and hence is finite. It follows that no plane $H \subset \mathbb{P}^{3}$ can be tangent to $S$ along a curve; in other words, every hyperplane section of $S$ is reduced. The same is thus true of the fibers $C_{H}$ of the fibration $\pi: S \rightarrow M$.

We may thus list the possible singular fibers of $\pi$ : they are

- a cubic with one, two or three nodes: that is, an irreducible nodal curve, the union of a line and a conic meeting transversely, or the union of three nonconcurrent lines. These are called fibers of type $\mathrm{I}_{b}$ with $b=1,2$ and 3 respectively;

- a cuspidal cubic, called a fiber of type II;

- the union of a line and a tangent conic, called a fiber of type III; or

- the union of three concurrent lines, called a fiber of type IV.

Note that fibers of type $\mathrm{I}_{b}$ correspond to poles of order $b$ of the $j$-function on $M$ associated to the elliptic fibration $S \rightarrow M$. By contrast, fibers of type II and IV correspond to zeroes of $j$-after base changes of order 6 and 3 respectively, we may replace the singular curve by an elliptic curve $\tilde{C}$ of $j$-invariant 0 - and fibers of type III correspond to points where $j=1728$ : after a base change of order 4 we may replace the singular curve by an elliptic curve $\tilde{C}$ of $j$-invariant 1728 . The monodromy action on the homology of the general fiber around a fiber of type $\mathrm{I}_{b}$ is thus given by the PicardLefschetz transformation, while in the case of fibers of type II, III and IV the monodromy is the just the action of the automorphism given by the base change on $\tilde{C}$. We list here these actions; see the original paper of Kodaira or the discussion in Barth-Peters-Van de Ven for details (cf. [1]). 


\begin{tabular}{c|c} 
type & monodromy \\
\hline $\mathrm{I}_{b}$ & $M_{\mathrm{I}_{b}}=\left(\begin{array}{ll}1 & b \\
0 & 1\end{array}\right)$ \\
\hline $\mathrm{II}$ & $M_{\mathrm{II}}=\left(\begin{array}{cc}1 & 1 \\
-1 & 0\end{array}\right)$ \\
\hline $\mathrm{III}$ & $M_{\mathrm{III}}=\left(\begin{array}{cc}0 & 1 \\
-1 & 0\end{array}\right)$ \\
\hline $\mathrm{IV}$ & $M_{\mathrm{IV}}=\left(\begin{array}{cc}0 & 1 \\
-1 & -1\end{array}\right)$
\end{tabular}

There is one constraint on the number of singular fibers $C_{H}$ : by a standard Euler characteristic calculation,

$$
\chi(S)=24=\sum_{H \in M} \chi\left(C_{H}\right) .
$$

The Euler characteristics of fibers of type $\mathrm{I}_{b}$, II, III and IV are $b$ (with $b=1,2$ or 3 in our case), 2, 3 and 4 respectively, giving a linear relation on the numbers of fibers of each type. In particular, we see that there must be at least 6 singular fibers $C_{H}$.

A related issue is the count of curves $C_{H}$ that are not transverse to $L$. Given that the divisors $D_{H}$ cut out on $L$ by the curves $C_{H}$ form a pencil of degree 3, it follows by Riemann-Hurwitz that there must be a total of 4 branch points, counting multiplicity: that is, either two curves $C_{H}$ having a point of intersection multiplicity 3 with $L$, one such curve and two others having a double point of intersection with $L$, or four curves $C_{H}$ having a double point of intersection with $L$. Moreover, note that the monodromy on the points $p_{i}$ around a curve $C_{H_{0}}$ having a point $p$ of intersection multiplicity 2 (respectively, 3 ) with $L$ is necessarily cyclic of order exactly 2 (respectively, 3 ). It follows that if the points $p$ of $C_{H} \cap L$ differ from each other by torsion in $\operatorname{Pic}\left(C_{H}\right)$ for general $H$, then since sections of an elliptic fibration that differ by torsion in the general fiber can intersect only at singular points of fibers, the multiple point $p$ of intersection of $C_{H_{0}}$ with $L$ must be a singular point of $C_{H_{0}}$. 
On the other hand, since there can be at most four fibers $C_{H}$ having multiple points of intersection with $L$, and each can contribute at most 4 to the Euler characteristic, we may draw one conclusion in particular that will turn out to be vital to the following analysis: that there must be singular fibers $C_{H}$ that intersect $L$ transversely.

\subsection{Analysis of the points of $C_{H} \cap L$}

We now ask the crucial question: is it possible that for general planes $H$ containing $L$, the points of intersection of $C_{H}$ with $L$ are all rationally related to the class $\mathcal{O}_{C_{H}}(1) \in \operatorname{Pic}\left(C_{H}\right)$ ? For the remainder of this subsection, then, we will assume that this is the case, and see what conclusions we may draw from it.

So: let $H$ be a general plane containing $L$, and let $p_{1}, p_{2}$ and $p_{3}$ be the three points on intersection of $C_{H}$ with $L$. Assume that some multiple of $p_{i}$ is linearly equivalent to a multiple of the hyperplane section of $C_{H}$, and let $n$ be the smallest positive integer such that

$$
3 n \cdot p_{i} \sim \mathcal{O}_{C_{H}}(n) .
$$

Note that since the monodromy on the three points $p_{i}$ as $H$ varies is at least transitive, this hypothesis will hold for one $p_{i}$ if and only if it holds for all three, and the value of $n$ will be the same for all three. In this case, the pairwise differences

$$
\alpha_{i, j}=p_{i}-p_{j} \in \operatorname{Pic}^{0}\left(C_{H}\right) i \neq j
$$

are torsion; we let $m$ be their order (again, since the monodromy is transitive on the three pairs $\left\{ \pm \alpha_{i, j}\right\}=\left\{\alpha_{i, j}, \alpha_{j, i}\right\}$, the value of $m$ will be the same for all $i$ and $j$ ). Note that the classes $\alpha_{i, j}$ are all nonzero, but need not be distinct: if $m=2$, of course, we have $\alpha_{i, j}=\alpha_{j, i}$, while if $m=3$ we could have $\alpha_{1,2}=\alpha_{2,3}=\alpha_{3,1}$. If $m>3$, however, we can see from the transitivity of the monodromy and the fact that $\alpha_{1,2}+\alpha_{2,3}+\alpha_{3,1}=0$ that they must be distinct.

Note finally that if $m \neq 2$, the monodromy on the points $p_{i}$ must be cyclic, rather than the symmetric group $S_{3}$ : a transformation fixing $p_{1}$, for example, and exchanging $p_{2}$ and $p_{3}$, would exchange $\alpha_{1,2}$ and $\alpha_{1,3}$ and send $\alpha_{2,3}$ to $-\alpha_{2,3}$; given that $\alpha_{1,2}-\alpha_{1,3}+\alpha_{2,3}=0$, this implies $2 \alpha_{2,3}=0$. It 
follows in particular that in case $m \neq 2$, there are exactly two fibers $C_{H}$ not transverse to $L$, and each has a triple point of intersection with $L$.

To carry out the further analysis of the monodromy action on the points $p_{i}$ and the classes $\alpha_{i, j}$, we will consider in turn three potential cases: $m>3$, $m=3$ and $m=2$.

Case a: $m>3$. The crucial observation here is simple enough: it is that if $C_{H}$ is any fiber transverse to $L$, then the monodromy around $C_{H}$ acts trivially on the points $p_{i}$ and hence on the classes $\alpha_{i, j}$. Let us consider accordingly the fixed point sets of the various monodromy transformations listed above:

1. The transformation $M_{\mathrm{I}_{b}}$ visibly fixes a cyclic subgroup of the points of order $m$ in $\operatorname{Pic}^{0}\left(C_{H}\right)$, and fixes no other primitive point of order $m$.

2. $M_{\text {II }}$ has characteristic polynomial $p(\lambda)=\lambda^{2}-\lambda+1$, which does not vanish at 1 ; thus $M_{\mathrm{II}}$ does not fix any primitive points of order $m$.

3. $M_{\text {III }}$ has characteristic polynomial $p(\lambda)=\lambda^{2}+1$, and since we have assumed $m \neq 2$ this does not vanish at 1 ; thus $M_{\text {III }}$ does not fix any primitive points of order $m$.

4. $M_{\mathrm{IV}}$ has characteristic polynomial $p(\lambda)=\lambda^{2}+\lambda+1$, and since we have assumed $m \neq 3$ this does not vanish at 1 ; thus $M_{\mathrm{IV}}$ does not fix any primitive points of order $m$.

The conclusion is plain: all singular fibers $C_{H}$ transverse to $L$ must be of type $\mathrm{I}_{b}$; and since we have already seen that there must exist such fibers, we deduce further that the classes $\alpha_{i, j}$ lie in a cyclic subgroup $G \cong \mathbb{Z} / m$ of $\operatorname{Pic}^{0}\left(C_{H}\right)$.

We ask now what may be the singular fibers $C_{H}$ that are singular at a point of $L$. As we have seen, since $m \neq 2$ there are exactly two fibers $C_{H}$ not transverse to $L$, and each has a triple point of intersection with $L$. For each such fiber, moreover, the subgroup $G \subset \operatorname{Pic}^{0}\left(C_{H}\right)$ spanned by (any one of) the $\alpha_{i, j}$ is an eigenspace for the action of the monodromy on the points of order $m$ in $\operatorname{Pic}^{0}\left(C_{H}\right)$. Now, the monodromy action $M_{\mathrm{I}_{b}}$ associated to a singular fiber of type $\mathrm{I}_{b}$ has only one eigenspace, with eigenvalue 1 . But the monodromy on the points $p_{i}$ is necessarily nontrivial and cyclic, and since 
$m \neq 3$ it follows that the action on the classes $\alpha_{i, j}$ is nontrivial. Thus a fiber of type $\mathrm{I}_{b}$ singular at a point of $L$ cannot occur.

Next, suppose that we have a singular fiber of type II. As we saw, the monodromy $M_{\text {II }}$ has characteristic polynomial $p(\lambda)=\lambda^{2}-\lambda+1$. Suppose $\lambda$ is a root of this polynomial mod $(m)$. Then we have

$$
\lambda^{2} \equiv \lambda-1 \bmod m
$$

and hence

$$
\lambda^{3} \equiv \lambda^{2}-\lambda \equiv-1 \quad \bmod m \text {. }
$$

Alternatively, we can just multiply out and see that $M_{\mathrm{II}}^{3}=-1$. Either way, we see that $M_{\mathrm{II}}^{3}$ cannot fix any element of $\operatorname{Pic}^{0}\left(C_{H}\right)$ of order $m \neq 2$, and so no fiber of this type can occur.

A similar analysis shows that no fiber of type III can occur (or we could just observe that the union of a line and a tangent conic cannot have a point of intersection multiplicity 3 with $L$ ); and so we conclude in sum that every singular fiber $C_{H}$ transverse to $L$ must be of type $\mathrm{I}_{b}$; and every fiber not transverse to $L$ must be of type IV. In particular, we see that in this case $L$ must meet at least the 6 other lines of $S$ comprising the two fibers of type IV.

We do not know of any examples of this case.

Case b: $m=3$. The analysis here is very similar to the preceding case, with one exception. To begin with, the same analysis shows that the fibers $C_{H}$ not transverse to $L$ must both be of type IV: none of other transformations $M_{\mathrm{I}}, M_{\mathrm{II}}$ and $M_{\mathrm{III}}$ has a three-cycle on the points of order 3 . One difference is that it is a priori possible that the classes $\alpha_{i, j}$ do not lie in a cyclic subgroup: this could be the case if every singular fiber $C_{H}$ transverse to $L$ is of type $\mathrm{I}_{3}$. But if there were $\delta$ such fibers, the formula for the Euler characteristic gives

$$
24=2 \cdot 4+\delta \cdot 3
$$

which has no solution. Thus the classes $\alpha_{i, j}$ lie in a cyclic subgroup, which means in turn that we must have $\alpha_{1,2}=\alpha_{2,3}=\alpha_{3,1}$. Thus in turn implies that

$$
3 p_{i} \sim p_{1}+p_{2}+p_{3} \sim \mathcal{O}_{C_{H}}(1)
$$


that is, $n=1$, or in other words all three points $p_{i}$ are flexes of $C_{H}$ for general $H$. Again, we do not know if this is possible. In any event, we see again that this case can occur only when six or more other lines of $S$ meet $L$.

Case c: $m=2$. This case gives us the least amount of control over the behavior of the singular fibers $C_{H}$ not transverse to $L$, but happily the most over the behavior of those that are. Very simply, in this case the classes $\alpha_{i, j}$ cannot lie in a cyclic subgroup: they must comprise all three classes of order 2 in $\operatorname{Pic}^{0}\left(C_{H}\right)$. It follows in turn that the monodromy associated to each fiber $C_{H}$ transverse to $L$ must be trivial on the points of order 2 in $\operatorname{Pic}^{0}\left(C_{H}\right)$, which says in turn that every singular fiber $C_{H}$ transverse to $L$ must be of type $\mathrm{I}_{2}$, that is, must consist of the union of a line and a conic. Now, as far as we can tell, the fibers $C_{H}$ not transverse to $L$ can be of type II, III or IV, but in any event the total contribution of such fibers to the Euler characteristic can be at most 8 (if we have either four fibers of type III or two of type IV). The remaining singular fibers must therefore contribute at least 16 to the Euler characteristic, which means we must have at least 8 fibers of type $\mathrm{I}_{2}$; in particular, $L$ must intersect at least 8 other lines of $S$.

In sum, we have established the

Theorem 4.1 Let $S \subset \mathbb{P}^{3}$ be a smooth quartic surface and $L \subset S$ a line in $\mathbb{P}^{3}$ contained in $S$; assume that $L$ does not meet six or more other lines contained in $S$. Let $n$ be any positive integer. For all but finitely many $p \in L$,

$$
3 n \cdot p \not \mathcal{O}_{C_{p}}(n)
$$

\section{Rational points on quartic surfaces}

To deduce part b) of Theorem 1.5 from the analysis carried out so far, we need one more ingredient. Briefly, Theorems 3.1 and 4.1 assure us (subject to their hypotheses) that for a very general point $p \in L(\mathbb{C})$ - that is, for all but countably many points $p \in L(\mathbb{C})$ - the point $p \in C_{p}$ is not rationally related to the hyperplane class in $\operatorname{Pic}\left(C_{p}\right)$. If such a point $p$ lies in $L(K)$, the cubic $C_{p}$ has positive rank over $K$, and hence a dense set of rational points. It seems reasonable to expect that for "most" of the points $p \in L(K)$ this 
would be true. But there will be countably many points $p \in L(\overline{\mathbb{Q}})$ for which $p$ is rationally related to $\mathcal{O}_{C_{p}}(1)$ - for each $n$, there is a finite subset $\Phi_{n} \subset L$ $p \in L(\overline{\mathbb{Q}})$ such that $3 n \cdot p \sim \mathcal{O}_{C_{p}}(n)$ in $\operatorname{Pic}^{0}\left(C_{p}\right)$ - and it is still a logical possibility, if not a plausible one, that all the points of $L(K)$ lie in this set.

There are two ways of eliminating this possibility. The first is to invoke an extremely powerful theorem due to Merel [23]:

Theorem 5.1 (Merel) Let $K$ be any number field. There is an integer $n_{0}=n_{0}(K)$ such that no elliptic curve defined over $K$ has a $K$-rational point of order $n>n_{0}$.

This theorem assures us that for $n>n_{0}$, the subset $\Phi_{n}$ is disjoint from $L(K)$; so that for all but finitely many $p \in L(K)$ the point $p$ will not be rationally related to $\mathcal{O}_{C_{p}}(1)$ in $\operatorname{Pic}\left(C_{p}\right)$.

A second way to arrive at this fact uses a theorem that is easier to prove, if less simple to state. Suppose that $E \rightarrow B$ is any family of elliptic curves and $\sigma$ a section of $E \rightarrow B$, both defined over a number field $K$. For each point $t \in B$, let $h_{B}(t)$ be the height of $t$ (relative to any divisor of degree 1 on $B), h\left(\sigma_{t}\right)$ the canonical height of the value $\sigma_{t}$ of the section in the fiber $E_{t}$ of the family over $t$, and $h(\sigma)$ the canonical height of the section $\sigma$ in the fiber of $E$ over the generic point of $B$. We have then the following theorem of Dem'janenko [12], Manin [22] and Silverman ([30], Theorem B, p. 197):

\section{Theorem 5.2}

$$
\lim _{h_{B}(t) \rightarrow \infty} \frac{h\left(\sigma_{t}\right)}{h_{B}(t)}=h(\sigma) .
$$

In particular, to say that $\sigma$ is not a torsion point in the fiber of $E$ over the generic point $\eta$ of $B$ is to say that $h(\sigma)>0$, and we can deduce the

Corollary 5.3 If $\sigma_{\eta}$ is not a torsion point in the fiber $E_{\eta}$ of $E$ over the generic point $\eta \in B$, then there are only finitely points $t \in B(K)$ such that $\sigma_{t}$ is a torsion point in $E_{t}$.

Applying this to the family $T \rightarrow L$ of elliptic curves introduced in section 3 (with the origin given by the tautologous section $\Sigma$ and the section $\sigma$ given by the divisor class $\mathcal{O}_{C_{p}}(1)$ in each fiber, we deduce again that (subject 
to the hypotheses of Theorem 4.1) for all but finitely many $p \in L(K)$ the point $p$ will not be rationally related to $\mathcal{O}_{C_{p}}(1)$ in $\operatorname{Pic}\left(C_{p}\right)$.

In fact, a weaker version, due to Néron would suffice - he proved that there are infinitely many points in $B(K)$ with fiber containing infinitely many $K$-rational points (see Serre's book [29], p. 153).

Now, suppose $S \subset \mathbb{P}^{3}$ is a smooth quartic surface defined over a number field $K$ and $L \subset S$ a line in $\mathbb{P}^{3}$ contained in $S$, likewise defined over $K$; assume that $L$ does not meet six or more other lines contained in $S$ (not necessarily defined over $K)$. For each point $p \in L(K)$ and for each integer $n$ there are unique points $q_{n}$ and $r_{n} \in C_{p}$ such that

$$
q_{n}+(3 n-1) \cdot p \sim \mathcal{O}_{C_{p}}(n)
$$

and

$$
-r_{n}+(3 n+1) \cdot p \sim \mathcal{O}_{C_{p}}(n),
$$

also defined over $K$. Moreover, for all but finitely many $p \in L(K)$ we have

$$
3 n \cdot p \not \mathcal{O}_{C_{p}}(n)
$$

for every $n$, so that these points are all distinct. We have, accordingly, an infinite collection of $K$-rational points on $C_{p}$, so that $C_{p}$ is contained in the Zariski closure of $S(K)$; since this is true of infinitely many curves $C_{p}$, it follows that the Zariski closure of $S(K)$ is all of $S$.

As for the proof of part a) of Theorem 1.5, given part b) this requires only one further trick, and it's a relatively simple one. This is expressed in the

Lemma 5.4 Let $S \subset \mathbb{P}^{3}$ be a smooth quartic surface and $L, L^{\prime}$ and $L^{\prime \prime} \subset S$ three lines in $\mathbb{P}^{3}$ contained in $S$; assume that $L$ does not meet either $L^{\prime}$ or $L^{\prime \prime}$, but that $L^{\prime}$ and $L^{\prime \prime}$ do meet. For each plane $H \supset L$ containing $L$, let $q_{H}=C_{H} \cap L^{\prime}$ and $r_{H}=C_{H} \cap L^{\prime \prime}$. If $H \supset L$ is a general plane containing $L$, then the difference $q_{H}-r_{H}$ is not torsion in $\operatorname{Pic}^{0}\left(C_{H}\right)$.

Proof. This is easy. Note first that since $L$ doesn't meet $L^{\prime}$ and $L^{\prime \prime}$, no plane $H$ containing $L$ can be the tangent plane to $S$ at any point of $L^{\prime}$ or $L^{\prime \prime}$; in other words, $q_{H}$ and $r_{H}$ are smooth points of the curve $C_{H}$ for all $H$. 
Now let $T$ be as in the proof of Theorem 3.1. The assignment to each point $p \in L$ of the points $q_{p}$ and $r_{p} \in C_{p}$ gives sections of the map $T \rightarrow L$, which we claim do not differ by a translation of finite order in the general fiber $C_{p}$. But two sections of an elliptic fibration that differ by torsion in the general fiber can intersect only at singular points of fibers, and so we are done.

Proof of part a) of Theorem 1.5. Now suppose we have a quartic surface $S$ and a line $L \subset S$. If fewer than six other lines of $S$ meet $L$, we may apply part b) of Theorem 1.5 to conclude that the points of $S$ rational over the field of definition of $L$ are Zariski dense. Suppose conversely that every line of $S$ meets at least six other lines of $S$. We claim in that case that $S$ must contain a configuration of lines as in Lemma 5.4. To see this, start with any line $L_{0} \subset S$. Since no more than four lines on $S$ can pass through a single point of $S$, we have to consider only two possibilities:

Case 1: three pairwise skew lines $L_{1}, L_{2}$ and $L_{3} \subset S$ meet $L_{0}$. In this case, let $M_{1}, \ldots, M_{5} \subset S$ be five other lines meeting $L_{1}$ (in addition to $L_{0}$ ). If any one of them fails to meet both $L_{2}$ and $L_{3}$, we are done: if $M_{i}$ fails to meet $L_{j}$, we take for our configuration $L=L_{j}, L^{\prime}=L_{1}$ and $L^{\prime \prime}=M_{i}$. On the other hand, if all six lines $L_{0}, M_{1}, \ldots, M_{5}$ meet all three lines $L_{1}, L_{2}$ and $L_{3}$, they must all lie on the unique quadric surface $Q \subset \mathbb{P}^{3}$ containing $L_{1}, L_{2}$ and $L_{3}$ - but then we have nine lines in $Q \cap S$, contradicting Bézout (among others).

Case 2: two triples of concurrent lines $\left\{L_{1}, L_{2}, L_{3}\right\}$ and $\left\{M_{1}, M_{2}, M_{3}\right\}$ meet $L_{0}$. Even easier: just let $N$ be any line meeting $L_{1}$ and skew to $L_{0} . N$ can't meet all three lines $M_{1}, M_{2}$ and $M_{3}$ (it would be coplanar with them and hence meet $L_{0}$ ); if it misses $M_{i}$ we take $L=M_{i}, L^{\prime}=L_{1}$ and $L^{\prime \prime}=N$.

To complete the proof of Theorem 1.5, suppose now that $L, L^{\prime}$ and $L^{\prime \prime} \subset S$ are a configuration as in Lemma 5.4; let $K^{\prime}$ be any field over which $S$ and all three lines are defined. For each plane $H$ containing $L$ and each integer $n$ there is a unique point $x_{n} \in C_{H}$ such that

$$
x_{n}+n \cdot q_{H} \sim(n+1) \cdot r_{H},
$$

also defined over $K^{\prime}$. Moreover, by Merel's theorem and Lemma 5.4 (or also by Silverman's theorem), for all but finitely many $H$ these points are all 
distinct. We have thus for infinitely many $H$ an infinite collection of $K^{\prime}$ -

rational points on $C_{H}$, and once more it follows that the Zariski closure of $S\left(K^{\prime}\right)$ is all of $S$.

\section{An example: the Fermat quartic}

In light of the analysis above, it might seem unlikely that there is any quartic surface $S$ and line $L$ such that for general planes $H$ containing $L$, the points of $C_{H} \cap L$ are all rationally related to the hyperplane class in $\operatorname{Pic}\left(C_{H}\right)$. In fact, however, it does occur: we will describe here the unique example we know of, the Fermat surface. See also the analysis of Piatetski-Shapiro and Shafarevitch in [28].

To begin with, we take $S \subset \mathbb{P}^{3}$ the quartic given by the equation

$$
X^{4}-Y^{4}+Z^{4}-W^{4}=0
$$

and $L$ the line

$$
X=Y \text { and } Z=W .
$$

Any plane containing $L$, other than the plane $Z=W$, can be realized as the span of $L$ and a third point of the form $[a,-a, 1,-1]$ for some scalar $a$; that is, the plane given parametrically by

$$
[U, V, T] \longmapsto[U+a T, U-a T, V+T, V-T]
$$

Restricting the equation of $S$ to $H$ gives the equation

$$
(U+a T)^{4}-(U-a T)^{4}+(V+T)^{4}-(V-T)^{4}=8 a U^{3} T+8 a^{3} U T^{3}+8 V^{3} T+8 V T^{3}
$$

so the equation of $C_{H}$ is simply

$$
F_{a}(U, V, T)=a U^{3}+a^{3} U T^{2}+V^{3}+V T^{2}=0 .
$$

The points of $C_{H} \cap L$ are given by the further equation $T=0$; that is, they are the points

$$
[U, V, T]=[1, b, 0]
$$

where $b^{3}=a$. Note that the monodromy on these as $a$ varies is cyclic. 
What are the singular fibers $C_{H}$ of the fibration $S \rightarrow M$ ? The plane $Z=$ $W$ corresponding to $a=\infty$ is certainly one, consisting of three concurrent lines meeting a point of $L$; the plane $X=Y$ corresponding to $a=0$ is another singular fiber of type IV. To find the remaining ones we have simply to write out the partial derivatives of $F_{a}(U, V, T)$ and equate them all to 0 ; the equations

$$
3 a U^{2}+a^{3} T^{2}=3 V^{2}+T^{2}=2 V T+2 a^{3} U T=0
$$

together imply that $a^{4}= \pm 1$, and that

$$
U=\frac{1}{\sqrt{-3}} T \text { and } \quad V=\frac{a}{\sqrt{-3}} T
$$

There are thus 8 singular fibers apart from $a=0$ and $a=\infty$, each having two singular points (that is, consisting of a line and conic).

We claim now that the three points $p_{i}$ of intersection of $C_{H}$ with $L$ differ by torsion of order 2 , and each satisfies $6 \cdot p_{i} \sim \mathcal{O}_{C_{H}}(2)$. The second statement follows from the first, given that $p_{1}+p_{2}+p_{3} \sim \mathcal{O}_{C_{H}}(1)$, and the first is readily checked: we simply observe that two points $p$ and $q$ on a plane cubic curve $C$ differ by torsion of order 2 if and only if the point of intersection $\mathbb{T}_{p} C \cap \mathbb{T}_{q} C$ of the tangent lines to $C$ at $p$ and $q$ lies on $C$. Now, the equation of the tangent line to $C_{H}$ at a point $[\mu, \nu, \tau]$ is

$$
\left(3 a \mu^{2}+a^{3} \tau^{2}\right) \cdot U+\left(3 \nu^{2}+\tau^{2}\right) \cdot V+\left(2 \nu \tau+2 a^{3} \mu \tau\right) \cdot T=0
$$

and at the point $[1, b, 0]$ for some cube root $b$ of $a$ this is

$$
-3 b^{3} \cdot U+3 b^{2} \cdot V=0 .
$$

For any two distinct cube roots $b$ of $a$, the resulting linear forms in $U$ and $V$ are independent, so that the point of intersection of any two of the tangent lines to $C_{H}$ at the points of $C_{H} \cap L$ is just

$$
[U, V, T]=[0,0,1]
$$

which is a point of $C_{H}$. 
Again, we don't know of any other examples of a quartic surface $S$ and a line $L \subset S$ such that for general planes $H$ containing $L$ the points of $C_{H} \cap L$ are all rationally related to the hyperplane class in $\operatorname{Pic}\left(C_{H}\right)$, nor do we know any examples at all where the points differ from each other by torsion of order greater than 2 .

\section{Quartic threefolds}

We would now like to use Theorem 1.5 to deduce Theorem 1.4. This is relatively simple: we just have to check that if $X \subset \mathbb{P}^{n}$ is any smooth quartic hypersurface and $L \subset X$ any line, then for a general 3-plane $\mathbb{P}^{3} \subset \mathbb{P}^{n}$ containing $L$, the surface $S=X \cap \mathbb{P}^{3}$ and the line $L$ satisfy the hypotheses of part b) of Theorem 1.5. It's enough to do this in case $n=4$, and it requires only a straightforward geometric argument.

We start with a basic fact:

Lemma 7.1 If $X \subset \mathbb{P}^{4}$ is a smooth quartic hypersurface, the Fano variety $F_{1}(X) \subset \mathbb{G}(1,4)$ of lines on $X$ has pure dimension one.

Proof. To begin with, the homogeneous quartic polynomial $F \in \operatorname{Sym}^{5}\left(\mathbb{C}^{5}\right)$ on $\mathbb{P}^{4}$ defining $X$ gives rise to a section $\tau_{F}$ of the fifth symmetric power $\operatorname{Sym}^{5}\left(S^{*}\right)$ of the dual of the universal subbundle $S$ on $\mathbb{G}(1,4)$, and the zero locus of this section is the Fano scheme $F_{1}(X)$. This shows that it has dimension at least 1 everywhere, and since the top Chern class $c_{5}\left(\operatorname{Sym}^{5} S^{*}\right)=$ $320 \sigma_{3,2} \neq 0$ that it is nonempty; it remains only to see that it cannot be twoor higher-dimensional.

To do this, let

$$
\Phi=\mathbb{P}\left(\left.S\right|_{F_{1}(X)}\right)=\{(L, p): p \in L\} \subset F_{1}(X) \times X
$$

be the universal projective line bundle over the Fano variety $F_{1}(X) \subset \mathbb{G}(1,4)$, and $\rho: \Phi \rightarrow X \subset \mathbb{P}^{4}$ the projection map. The tangent space to $F_{1}(X)$ at a point $L \in F_{1}(X)$ may be identified with the space of section $H^{0}\left(L, N_{L / X}\right)$; and in these terms, at a general point $(L, p) \in \Phi$ the image of the differential $d \rho_{(L, p)}: \mathbb{T}_{L} F_{1}(X) \rightarrow \mathbb{T}_{p} X \bmod \mathbb{T}_{p} L$ is simply the image of the map $H^{0}\left(L, N_{L / X}\right) \rightarrow\left(N_{L / X}\right)_{p}=\mathbb{T}_{p} X / \mathbb{T}_{p} L$ given by evaluation at $p$. Since $N_{L / X}$ 
has exactly one summand of nonnegative degree, this image is always onedimensional $\left(\bmod \mathbb{T}_{p} L\right)$, and so we may conclude that the image of the map $\rho: \Phi \rightarrow X$-that is, the union of the lines on $X$-is always exactly 2dimensional. But $X$ contains no 2-planes, and no surface in projective space other than a 2-plane may contain $\infty^{2}$ lines, so we may conclude that $X$ contains only $\infty^{1}$ lines.

On the basis of a naive dimension count, we would expect the map $\rho$ from the two-dimensional variety $\Phi$ to the threefold $X$ to have a one-dimensional double point locus, that is, $\infty^{1}$ pairs of distinct lines $L, L^{\prime} \subset X$ that meet; we'd thus expect that each line of $X$ would meet finitely many others. (We'll calculate the number in just a moment.) Accordingly, we'll call a line $L \subset X$ exceptional if it meets infinitely many other lines of $X$. We will denote by $F_{1}^{e}(X) \subset F_{1}(X)$ the locus of exceptional lines.

By a straightforward dimension count, a general quartic threefold $X$ has no exceptional lines, and a general $X$ containing an exceptional line will contain only finitely many; in particular, it will contain nonexceptional lines as well. Our situation is that we're able to apply Theorem 1.5 directly to the surface $S=H \cap X$ where $H$ is a general hyperplane section containing a nonexceptional line $L$, and so our concern is whether an arbitrary $X$ may contain only exceptional lines. In fact, this is possible: the Fermat quartic is one example (we don't know any other examples). What we want to do, accordingly, is to say as much as we can about quartic threefolds that have positive-dimensional families of exceptional lines.

So: let $X$ be a smooth quartic threefold, and $\Psi \subset F_{1}^{e}(X)$ an irreducible component of the Fano variety of lines on $X$ consisting entirely of exceptional lines. For each line $L \in \Psi$, the locus of lines meeting $L$ will contain one or more irreducible components of $F_{1}(X)$, and so we must have one of the following two situations:

1. There are two irreducible components $\Psi, \Psi^{\prime} \subset F_{1}(X)$ such that every pair of lines $L \in \Psi$ and $L^{\prime} \in \Psi^{\prime}$ meet; or

2. There is an irreducible component $\Psi \subset F_{1}(X)$ such that every pair of lines $L, L^{\prime} \in \Psi$ meet.

In the first case, the surface $S \subset X$ swept out by the lines of $\Psi$ has two rulings by lines; but the only surface in projective space with two rulings by lines is a quadric surface in $\mathbb{P}^{3}$, and since $\operatorname{Pic}(X)=\mathbb{Z}\left\langle\mathcal{O}_{X}(1)\right\rangle, X$ contains 
no such surfaces; thus the first case cannot occur. In the second case, let $L, L^{\prime} \in \Psi$ be two general lines, meeting at a point $p$. A third general line $L^{\prime \prime} \in \Psi$ must meet both $L$ and $L^{\prime}$; if it doesn't pass through $p$ it must lie in the plane spanned by $L$ and $L^{\prime}$, and so this plane would have to be contained in $X$. Since $X$ contains to 2-planes, we conclude that all the lines $L \in \Psi$ have a common point $p$. It follows in particular that all the lines $L \in \Psi$ are contained in the tangent plane $\mathbb{T}_{p} X$, and hence that $X \cap \mathbb{T}_{p} X$ is simply the cone with vertex $p$ over an irreducible plane quartic curve. Finally, since $X$ is smooth the Gauss map $\mathcal{G}$ cannot be constant on any curve of $X$; thus $X \cap \mathbb{T}_{p} X$ can have at most isolated singularities and hence the curve $C$ must be smooth. We have thus established the

Proposition 7.2 Let $X$ be a smooth quartic threefold, and $\Psi \subset F_{1}^{e}(X)$ an irreducible component of the Fano variety of lines on $X$ consisting entirely of exceptional lines. Then there is a point $p \in X$ such that $X \cap \mathbb{T}_{p} X$ is the cone with vertex $p$ over a smooth plane quartic curve, and $\Psi$ is simply the ruling of this cone. In particular, $\Psi \subset \mathbb{G}(1,4) \subset \mathbb{P}^{9}$ has as underlying reduced scheme a smooth plane quartic curve.

It's a nice exercise to check directly that in this case the component $\Psi$ of the Fano scheme $F_{1}(X)$ has multiplicity 2. Since by the proof of Lemma 7.1 the Fano scheme is a curve of degree 320 in $\mathbb{G}(1,4) \subset \mathbb{P}^{9}$, it follows that a quartic threefold $X$ will contain only exceptional lines if and only if it has exactly 40 hyperplane sections consisting of cones over quartic plane curves. Again, this is the case for the Fermat quartic; we don't know if there are others.

In any event, Theorem 1.4 now follows readily from Theorem 1.5. Let $X \subset \mathbb{P}^{4}$ be any quartic threefold defined over a field $K$. Suppose first that $X$ contains a non-exceptional line $L$; say $L$ is defined over a field $K^{\prime} \supset K$. Then for a general hyperplane $H \cong \mathbb{P}^{3} \subset \mathbb{P}^{4}$ containing $L$, the surface $S_{H}=X \cap H$ will contain no other lines meeting $L$, and by part b) of Theorem 1.5 we may deduce that $S_{H}\left(K^{\prime}\right)$ is Zariski dense in $S_{H}$; hence $X\left(K^{\prime}\right)$ is Zariski dense in $X$.

If on the other hand every line of $X$ is exceptional, then by Proposition 7.2 the Fano variety of lines on $X$ will consist of a union of curves supported on plane quartic curves. If $L \subset X$ is a line corresponding to a general point $[L] \in \Psi \subset F_{1}(X)$ of a component $\Psi$ of $F_{1}(X)$ - in particular, if it is not a 
point of intersection of $\Psi$ with another component of $F_{1}(X)$ - then $L$ can meet only finitely many lines $L^{\prime} \subset X$ corresponding to points $\left[L^{\prime}\right] \notin \Psi$. It follows that for a general hyperplane $H \cong \mathbb{P}^{3} \subset \mathbb{P}^{4}$ containing $L$, the surface $S_{H}=X \cap H$ will contain exactly three other lines meeting $L$; so once again the hypothesis of part b) of Theorem 1.5 is satisfied, and we conclude that the points of $X$ rational over the field of definition of $L$ are Zariski dense.

\section{Other elliptic surfaces}

As suggested in section 3.1, the approach via the calculation of intersection numbers in the Néron-Severi group of an associated surface works in substantially greater generality. Specifically, we will prove

Theorem 8.1 Let $S$ be any smooth irrational surface and $\pi: S \rightarrow \mathbb{P}^{1}$ an elliptic fibration all of whose fibers are irreducible; for $\lambda \in \mathbb{P}^{1}$, let $E_{\lambda}=\pi^{-1}(\lambda)$ be the fiber of $S \rightarrow \mathbb{P}^{1}$ over $\lambda$. Let $C \subset S$ be any smooth rational or elliptic curve, of degree $m \geq 2$ over $\mathbb{P}^{1}$ and $n \neq 0$ any integer. Then for general $\lambda \in \mathbb{P}^{1}$ and any $p \in E_{\lambda} \cap C$,

$$
n m \cdot p \not \mathcal{O}_{E_{\lambda}} \otimes \mathcal{O}_{S}(n \cdot C)
$$

As before, we may deduce from this immediately the

Corollary 8.2 Let $S$ be any smooth surface, $\pi: S \rightarrow \mathbb{P}^{1}$ an elliptic fibration with irreducible fibers as in Theorem 8.1.

a) Let $C \subset S$ be a smooth rational curve. Assume that $S, \pi$ and $C$ are defined over a field $K$ and that $C$ is rational over $K$. Then the set $S(K)$ of $K$-rational points of $S$ is Zariski dense.

b) Now let $C \subset S$ be a smooth curve of genus 1 , and assume that $S, \pi$ and $C$ are defined over a field $K$. Then there is a finite extension $K^{\prime}$ of $K$ such that the set $S\left(K^{\prime}\right)$ of $K^{\prime}$-rational points of $S$ is Zariski dense.

Part a) of Corollary 8.2 follows from Theorem 8.1 as before: we see that for all but finitely many points $p \in C(K)$, the fiber of $S \rightarrow \mathbb{P}^{1}$ over $\pi(p)$ has infinitely many rational points. As for part b), we have to make an extension of our ground field $K$ simply to ensure that the curve $C$ has infinitely many 
rational points, and then the argument proceeds as before. Of course we can drop the hypothesis in Theorem 8.1 that $S$ is irrational.

Proof of Theorem 8.1. The proof is analogous to that of Theorem 3.1. We begin by making a base change: we let $T$ be the incidence correspondence

$$
T=\left\{(p, q): q \in E_{\pi(p)}\right\} \subset C \times S .
$$

As before, $T$ is the fiber product

$$
T=C \times_{\mathbb{P}^{1}} S
$$

in other words, $T \rightarrow C$ is the fibration obtained by applying the base change $\left.\pi\right|_{C}: C \rightarrow \mathbb{P}^{1}$ to the fibration $S \rightarrow \mathbb{P}^{1}$. In particular, $T$ is an $m$-sheeted cover of $S$, branched over the union of the fibers $E_{\lambda}$ of $S \rightarrow \mathbb{P}^{1}$ such that $E_{\lambda}$ is tangent to $C$. Again, $T$ will have at worst isolated singularities, since by the hypothesis that $S$ is smooth and all fibers of $\pi$ are irreducible it follows that all fibers are reduced as well. The surface $T$ is also normal since it is regular in codimension one and since it is locally defined by one equation in the smooth irreducible variety $C \times X$.

Note that $T \rightarrow C$ has a tautologous section

$$
\Sigma=\{(p, p): p \in C\} \subset T .
$$

As a divisor, the pullback $\nu^{*}(C)$ of the curve $C$ under the $m$-sheeted covering $\nu: T \rightarrow S$ is thus a sum

$$
\nu^{*}(C)=\Sigma+R
$$

with $R \subset T$ flat of degree $m-1$ over $C$. As before, since the general fiber of $C$ over $\mathbb{P}^{1}$ is reduced, $R$ does not contain $\Sigma$.

Now let $\phi \in A_{1}(T)$ be the class of a fiber of $T \rightarrow C, \sigma$ the class of the section $\Sigma$ and $\rho$ the class of $R$. The key ingredient in our proof is the

Lemma 8.3 The classes $\sigma, \rho$ and $\phi \in A_{1}(T)$ are independent in the group $A_{1}(T)$ of Weil divisors mod linear equivalence on $T$. 
Proof. We calculate the matrix of intersection products of the classes $\sigma, \rho$ and $\phi \in A_{1}(T)$. Three of these numbers are readily calculated. To begin with, $\phi$ is the class of a fiber of the map $T \rightarrow L$, so of course $\phi^{2}=0$; and since $\Sigma$ and $R$ meet each fiber in 1 and $m-1$ points respectively, we have $(\phi \cdot \sigma)=1$ and $(\phi \cdot \rho)=m-1$.

As before, we don't know anything about $c=(\sigma \cdot \rho)$ except that it is positive (if $T$ were smooth, if would have to be $2 m-2$ ). Finally, let $-b$ be the self-intersection of the curve $C$ on $S$. By the hypothesis that $S$ is irrational, the canonical class $K_{S}$ is a nonnegative (rational) multiple of the class of a fiber of $S \rightarrow \mathbb{P}^{1}$, and so has nonnegative intersection with $C$; it follows that $b \geq 2$ if $C$ is rational, and $g \geq 0$ is $C$ has genus 1 .

Now, to calculate $\sigma^{2}$ and $\rho^{2}$, we use the relation

$$
\nu^{*} C=\Sigma+R .
$$

It follows that

$$
\sigma^{2}=\left(\sigma \cdot\left[\nu^{*} C\right]-\rho\right),
$$

and since, by the push-pull formula,

$$
\left(\Sigma \cdot \nu^{*} C\right)_{T}=\left(\nu_{*} \Sigma \cdot C\right)_{S}=(C \cdot C)_{S}=-b
$$

we have

$$
\sigma^{2}=-b-c .
$$

Similarly, from the same relation it follows that

$$
\rho^{2}=\left(\rho \cdot\left[\nu^{*} C\right]-\sigma\right) .
$$

By the push-pull formula,

$$
\left(R \cdot \nu^{*} C\right)_{T}=\left(\nu_{*} R \cdot C\right)_{S}=((m-1) C \cdot C)_{S}=-(m-1) b
$$

and so

$$
\rho^{2}=-(m-1) b-c .
$$

In sum, then, we have the following table of intersection products 


\begin{tabular}{c|c|c|c|} 
& $\phi$ & $\sigma$ & $\rho$ \\
\hline$\phi$ & 0 & 1 & $m-1$ \\
\hline$\sigma$ & 1 & $-b-c$ & $c$ \\
\hline$\rho$ & $m-1$ & $c$ & $-(m-1) b-c$ \\
\hline
\end{tabular}

The determinant of this matrix is

$$
c(m-1)+(m-1) b+c+c(m-1)+(b+c)(m-1)^{2}
$$

and since $b$ is nonnegative and $c$ positive we are done.

\section{References}

[1] W. Barth, C. Peters, A. van de Ven, Compact complex surfaces, Ergebnisse der Mathematik und ihrer Grenzgebiete, Springer-Verlag, BerlinNew York, (1984).

[2] A. Beauville, Variétés de Prym et jacobiennes intermédiaires, Ann. Sci. Ecole Norm. Sup. (4) 10, no. 3, (1977), 309-391.

[3] A. Beauville, Variétés rationnelles et unirationnelles, Algebraic geometry - open problems (Ravello, 1982), LN in Math. 997, Springer, BerlinNew York, (1983), 16-33.

[4] F. Bogomolov, Yu. Tschinkel, Density of rational points on Enriques surfaces, Math. Research Letters 5, (1998), 623-628.

[5] F. Bogomolov, Yu. Tschinkel, Density of rational points on elliptic K3 surfaces, alg-geom 9902092, (1999).

[6] F. Bogomolov, Yu. Tschinkel, On the density of rational points on elliptic fibrations, alg-geom 9811043, (1998).

[7] F. Campana, Connexité rationnelle des variétés de Fano, Ann. Sci. École Norm. Sup. (4) 25, (1992), no. 5, 539-545.

[8] C. H. Clemens, Double solids, Adv. Math. 47, (1983), 107-230. 
[9] A. Collino, Lines on quartic threefolds, J. London Math. Soc. (2) 19, (1979), 257-267.

[10] J.-L. Colliot-Thélène, A. N. Skorobogatov and P. Swinnerton-Dyer, Double fibres and double covers: paucity of rational points. Acta Arith. 79:2, (1997), 113-135.

[11] O. Debarre, Variétés de Fano, Séminaire Bourbaki, Vol. 1996/97. Astérisque No. 245, (1997), Exp. No. 827, 4, 197-221.

[12] V. A. Dem'janenko, Rational points of a class of algebraic curves, A.M.S. Translations (2) 66, (1968), 246-272.

[13] J. Franke, Yu. I. Manin, Yu. Tschinkel, Rational points of bounded height on Fano varieties, Invent. Math. 95, no. 2, (1989), 421-435.

[14] W. Fulton, Intersection theory, 2nd edition, Ergebnisse der Mathematik und ihrer Grenzgebiete (3), Springer-Verlag, Berlin-New York, (1998).

[15] V. A. Iskovskikh, On the rationality problem for conic bundles, Duke Math. J. 54, no. 2, (1987), 271-294.

[16] V.A. Iskovskikh, Fano threefolds. I, Izv. Akad. Nauk SSSR Ser. Mat. 41, no. 3 , (1977), 516-562.

[17] V.A. Iskovskikh, Fano threefolds. II, Izv. Akad. Nauk SSSR Ser. Mat. 42, no. 3, (1978), 506-549.

[18] V.A. Iskovskikh, Yu. I. Manin, Three dimensional quartics and counterexamples to Lüroth problem, Mat. Sbornik 86, (1971), 140-166.

[19] V. A. Iskovskikh, Yu. G. Prokhorov, Fano varieties. Algebraic geometry V, Encyclopaedia Math. Sci., 47, Springer, Berlin, (1999).

[20] J. Kollár, Y. Miyaoka, Sh. Mori, Rational curves on Fano varieties, In Classification of irregular varieties (Trento, 1990), LN in Math. 1515, Springer, Berlin, (1992), 100-105.

[21] Yu. I. Manin, Notes on the arithmetic of Fano threefolds, Compositio Math. 85, (1993), 37-55. 
[22] Yu. I. Manin, The p-torsion of elliptic curves is uniformly bounded, Izv. Akad. Nauk. SSSR. 33, (1969), 433-438.

[23] L. Merel, Bornes pour la torsion des courbes elliptiques sur les corps de nombres, Invent. Math. 124, no. 1-3, (1996), 437-449.

[24] Sh. Mori, Sh. Mukai, On Fano 3-folds with $B_{2} \geq 2$, Algebraic varieties and analytic varieties (Tokyo, 1981), Adv. Stud. Pure Math. 1, NorthHolland, Amsterdam-New York, (1983), 101-129.

[25] Sh. Mori, Sh. Mukai, Classification of Fano 3-folds with $B_{2} \geq 2$, Manuscripta Math. 36, no. 2, (1981/82), 147-162.

[26] J. P. Murre, Classification of Fano threefolds according to Fano and Iskovskikh, Algebraic threefolds (Varenna, 1981), LN in Math. 947, Springer, Berlin-New York, (1982), 35-92.

[27] J. Noguchi, A higher-dimensional analogue of Mordell's conjecture over function fields, Math. Ann. 258, (1981/82), no. 2, 207-212

[28] I. I. Piatetski-Shapiro, I. Shafarevitch, Torelli's theorem for algebraic surfaces of type K3, (Russian) Izv. Akad. Nauk SSSR 35, (1971), 530572

[29] J.-P. Serre, Lectures on the Mordell-Weil theorem, 2nd ed., Aspects of Mathematics, Viehweg, (1990).

[30] J. H. Silverman, Heights and the specialization map for families of abelian varieties, J. reine und angew. Math. 342, (1983), 197-211.

[31] P. Vojta, Diophantine approximation and value distribution theory, LN in Math. 1239, Springer, Berlin-New York, (1987). 